EOMmUn: Communication et organisation

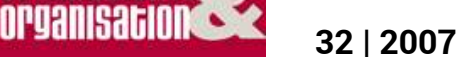

La ville dans tous les sens

Internet : vers une fragmentation de la ville?

Margot Beauchamps

\title{
OpenEdition
}

Journals

Édition électronique

URL : http://journals.openedition.org/communicationorganisation/340

DOI : 10.4000/communicationorganisation.340

ISSN : 1775-3546

Éditeur

Presses universitaires de Bordeaux

Édition imprimée

Date de publication : 1 décembre 2007

Pagination : 94-105

ISSN : 1168-5549

Référence électronique

Margot Beauchamps, «Internet : vers une fragmentation de la ville? », Communication et organisation

[En ligne], 32 | 2007, mis en ligne le 01 décembre 2010, consulté le 20 avril 2019. URL : http://

journals.openedition.org/communicationorganisation/340; DOI : 10.4000/

communicationorganisation.340

(c) Presses universitaires de Bordeaux 
Dossier : La ville dans tous les sens

\begin{abstract}
Résumé
Internet semble un moyen ubiquiste d'accès aux ressources traditionnellement offertes par la ville. Comme elle, il facilite l'interaction sociale, et donne accès à une infinité d'informations et de services, publics ou privés, marchands ou non. Sa large diffusion territoriale pourrait accréditer d'une part, l'idée de la mort de la ville, dont les ressources seraient désormais accessibles de partout, et d'autre part celle d'un effacement des disparités entre les différents quartiers de la ville par une égalité d'accès des différents groupes sociaux aux ressources de la société de l'information. Cet article cherche à montrer comment l'inégal accès des personnes à Internet, la diversité des pratiques communicationnelles, ainsi que l'action des opérateurs de réseaux, tendraient à accentuer la fragmentation sociale qui s'exprime dans la ville par la spécialisation sociale des quartiers.
\end{abstract}

Mots-clés

Internet, infrastructures, pratiques, quartiers, ségrégation, fragmentation urbaine, fracture numérique.

\begin{abstract}
Internet appears as an ubiquitous way of access to the resources traditionally offered by the city. As the city, Internet fosters social interaction and gives access to a wide variety of informations and services. Its large social and spatial diffusion could give credence to the thesis of the death of city which resources would now be accessible from anywhere and to the idea of vanishing disparities of urban areas thanks to an equal access for the different social groups to the resources of informations society. This article seeks to assess how the uneven access to the Internet, the wide diversity of Internet uses as well as the practices of the telecommunication networks' operators tend to reinforce the splintering of the city into socially specialized areas.
\end{abstract}

\title{
Key-Words
}

Internet, infrastructures, practices, neighborhood/area, segregation, splintering urbanism, digital divide.

Margot Beauchamps est doctorante en Géographie et Aménagement $\mathrm{du}$ territoire à l'Université Panthéon-Sorbonne - Paris I sous la direction de Gabriel Dupuy. Elle est rattachée au CRIA, Centre de recherche sur les Réseaux, l'Industrie et l'Aménagement. 
Internet : vers une fragmentation de la ville?

\section{Internet : vers une fragmentation de la ville ? Margot Beauchamps}

margot.beauchamps@univ-paris1.fr

Les Technologies de l'Information et de la Communication (TIC) modifient profondément le rapport des citadins à la ville. De plus en plus, les services publics et privés, les commerces sont restructurés pour s'orienter vers des clients et des usagers dont on suppose qu'ils sont internautes. On évolue vers une configuration de la ville dans laquelle les services, marchands ou non, les commerces sont conçus pour les usagers des télécommunications, créant ainsi une dépendance aux TIC. Trouver un logement, un emploi, communiquer avec les membres de son réseau social ou l'élargir, réaliser des démarches administratives, se tenir informé, se divertir sont des actions de la vie quotidienne qui nécessitent de plus en plus souvent d'utiliser Internet.

Parce qu'il permet d'accéder à une infinité de services, Internet a pu être présenté comme un moyen de «faire venir la ville à la campagne », voire de contribuer à la mort de la ville. En offrant un accès à «presque tout » de «n'importe où », Internet gommerait les disparités territoriales, rendant ainsi l'espace fongible. Parallèlement à ces visions parfois qualifiées d'utopiques, géographes, urbanistes, architectes ont montré que par la matérialité de leurs infrastructures, mais aussi par les usages qui en sont faits, les TIC sont surtout urbaines. (Dupuy 1991, Picon 2007). Comme la ville, ce « géotype de substance sociétale fondé sur la coprésence » (Lévy Lussault 2003, p. 988), Internet maximise les opportunités d'interaction sociale, certes médiatisées par l'écran d'ordinateur. Pour autant, Internet ne se substitue pas à la ville. Cet article propose de montrer que loin de réaliser l'isotropie généralisée de l'espace, le développement d'Internet dans la ville contribue à une différenciation des quartiers.

\section{Un accès inégal aux TIC}

Si Internet permet d'accéder à une immense diversité de services, son inégale diffusion exclut une partie de la population des multiples opportunités qu'il offre. Des freins financiers ou culturels s'exercent pour limiter l'usage d'Internet parmi certaines franges de la société. Les équipements en outils de télécommunication et les usages des TIC sont, en effet, fortement déterminés par l'appartenance à une catégorie sociale. La dernière enquête TIC de l'INSEE révèle qu'en proportion, on trouve quatre fois plus d'internautes - personnes ayant utilisé Internet au moins une fois au cours du dernier mois - chez les 
diplômés du supérieur ( $83 \%$ ) que chez les non diplômés (18\%), et trois fois plus chez les cadres $(90 \%)$ que chez les ouvriers $(34 \%)^{1}$ (Frydel 2006). Ces inégalités d'usage d'Internet s'expliquent en partie par des inégalités d'équipement. Les diplômés du supérieur sont, en proportion, près de quatre fois plus nombreux à posséder une connexion Internet à domicile que les non diplômés $(54 \%$ contre $15 \%)$.

Avec la baisse du coût des équipements, le facteur financier joue un rôle de moins en moins important, quoique non négligeable, dans l'explication de ces inégalités d'équipement. Selon l'INSEE, les ménages ne disposant pas de micro-ordinateur l'expliquent le plus souvent par une absence de besoin (pour la moitié des ménages), ensuite par le fait de ne pas savoir s'en servir (un cinquième des ménages), et seulement en troisième position, par le coût (un sixième des ménages) (Frydel 2006, Sautory 2007). Cependant, l'aspect financier joue un rôle plus déterminant dans le fait de ne pas disposer de connexion Internet à domicile, car, contrairement à l'achat d'un ordinateur qui est un investissement ponctuel, souscrire à un abonnement téléphonique ou à un abonnement à Internet implique de s'engager $^{2}$ à verser mensuellement une certaine somme pendant souvent plus d'un an. L'effet de substitution de la ligne de téléphone fixe par le téléphone portable, qui touche $27 \%$ des ménages à bas revenus, explique également ces inégalités d'accès à Internet à domicile, puisque disposer d'une ligne fixe est la plupart du temps nécessaire pour accéder à Internet. (Sautory 2007).

L'aspect financier n'explique pas à lui seul ces inégalités d'équipements et d'usages des TIC. Notons d'abord que si le coût de l'équipement peut limiter l'usage d'Internet à domicile, la multiplication des lieux publics d'accès à Internet (écoles, bibliothèques, cybercafé, centres sociaux, etc.) est une alternative pour ceux dont l'unique frein est d'ordre financier. Différentes études montrent enfin que les personnes non équipées à domicile ont plus souvent recours à leur réseau familial ou amical pour accéder à Internet qu'aux lieux d'accès publics (Frydel 2006). Plus difficiles à lever, des freins culturels s'exercent qui éloignent d'Internet certaines franges de la population. C'est notamment le cas des personnes illettrées, la technologie Internet reposant essentiellement sur l'écrit. Plus généralement, on a pu montrer une corrélation entre l'usage

\footnotetext{
${ }^{1}$ Les données ont été recueillies en octobre 2005.

${ }^{2}$ Ceci implique notamment que les personnes exclues du système bancaire ne peuvent souscrire à un abonnement Internet.
} 
d'Internet et certaines pratiques culturelles: lecture, cinéma, etc. (Alibert et al. 2005). Le capital social semble également jouer un rôle dans l'explication des inégalités d'usage d'Internet. L'effet de réseau ou «effet de club» (Dupuy, op. cit.) est au fondement même des communications de personne à personne (ou au sein d'une communauté) sur Internet : l'intérêt de posséder une adresse e-mail est nul si l'on ne connaît personne qui en possède une. Le réseau social peut constituer en outre une ressource dans laquelle on peut puiser de l'aide pour pallier les obstacles techniques à l'utilisation de ce média (DiMaggio et al. 2001).

La distinction entre ceux qui ont accès à Internet à domicile et les autres, ou encore entre les internautes ${ }^{3}$ et les autres permet d'identifier une première dimension de la "fracture numérique», entendue ici dans son sens le plus courant, celui d'une séparation entre les connectés et les non connectés. On verra plus loin les limites de cette définition de la fracture numérique qui sépare la société en deux, que ce soit du point de vue de l'équipement à domicile ou du point de vue de l'usage, ici renseigné de façon binaire : on utilise Internet ou on ne l'utilise pas. Ce premier constat, fortement relayé dans les discours politiques, permet toutefois d'observer que cette fracture numérique coïncide largement avec une autre fracture, la "fracture sociale ", notion qui a connu un succès politique égal : les TIC sont inégalement appropriées par les différentes catégories sociales. Or, géographie et sociologie urbaines ne cessent de constater l'inégale répartition des différentes catégories sociales dans l'espace urbain (Brun et Rhein 1994). Coûts fonciers, différentiel d'accessibilité, représentations mentales et pratiques associées aux différentes portions de la ville se combinent pour expliquer la différentiation sociale des quartiers de la ville. Les catégories sociales, qui s'approprient de manières différenciées Internet, se répartissent inégalement dans la ville. Des processus d'agrégation et de ségrégation se combinent pour différencier les quartiers de la ville selon l'appartenance sociale de leurs habitants, leurs ressources financières mais aussi leur âge ou encore leur capital culturel. Quels impacts sur la ville peut avoir l'inégale diffusion des TIC dans la société ?

\section{Un risque de fragmentation urbaine par les opérateurs de réseaux}

Constatant l'inégale diffusion des TIC parmi les différentes catégories sociales et, par là même, parmi les différents quartiers de la ville, les

\footnotetext{
${ }^{3}$ Selon la définition Insee : personne qui a utilisé Internet au moins une fois au cours du mois écoulé.
} 
opérateurs de télécommunication sont tentés de traiter distinctement les différents territoires urbains. Avec la libéralisation du secteur des télécommunications, la desserte des territoires urbains en infrastructures de télécommunication ne se fonde pas seulement sur des critères de densité humaine, mais aussi sur le niveau de solvabilité et d'appropriation des TIC des populations résidentes. On voit ici poindre le risque d'une fragmentation de la ville par les opérateurs de réseaux, l'écart se creusant entre les quartiers bénéficiant des effets positifs de la concurrence (baisse des prix, diversité de l'offre de services, niveaux de desserte et de débit élevés) et ceux, marqués par des faiblesses socio-économiques, délaissés par les opérateurs (Graham et Marvin 2001).

Les pouvoirs publics français se sont beaucoup félicités de la large diffusion qu'a connue ces dernières années 1'ADSL ${ }^{4}$. Cette technologie d'accès à Internet à haut débit permet d'utiliser l'ancien réseau de paire cuivrée du téléphone pour faire transiter des données numériques. Le succès commercial de l'ADSL auprès des consommateurs français a permis aux opérateurs d'étendre la couverture géographique du haut débit. Aujourd'hui, $98 \%$ de la population française réside dans une zone éligible à l'ADSL. Sans s'attarder sur les difficultés d'accès au haut débit que connaissent les $2 \%$ restant, principalement situés en zone rurale, qui seront les plus longs, car les plus coûteux à raccorder, on peut constater, avec Agnès Huet et Pascal Buléon, qu' « un différentiel perdure à la fois en débits et en qualité de service entre les zones couvertes par des réseaux haut débit et celles qui ne le sont pas et au sein même des zones disposant des réseaux dits "haut débit" » (Huet et Buléon 2007). En effet, au sein des zones couvertes par les réseaux haut débit, et notamment au sein d'une même agglomération, on observe une différence entre les lignes dégroupées (c'est-à-dire ouvertes à la concurrence) et celles qui ne le sont pas (pour lesquelles seul l'opérateur historique, France Télécom, offre ces services de connexion ADSL, à un prix supérieur à ceux des autres opérateurs). De plus, les débits offerts par l'ADSL étant fortement déterminés par la distance au central téléphonique, une même offre commerciale peut recouvrir un différentiel de débit - et même de service - très important. Une ligne éloignée du central sera connectée à un débit moins élevé, qui peut limiter les applications auxquelles l'abonné aura accès (difficulté d'accéder aux services de télévisions, par exemple).

${ }^{4}$ Asymmetric Digital Subscriber Line. 
L'essor attendu de la fibre optique, technologie d'accès à Internet à «très haut débit », illustre bien le risque de fragmentation territoriale par l'inégal déploiement des réseaux de télécommunication. À l'heure où les acteurs des TIC montrent leur inquiétude quant à la capacité de la technologie dominante d'accès à Internet (l'ADSL) de répondre aux exigences croissantes des utilisateurs d'Internet en terme de débit, les offres de raccordement à la fibre optique lancées par une poignée d'opérateurs concernent une infime partie des foyers français, résidant dans des quartiers choisis selon la structure sociale de leur population. Les travaux de déploiement de la fibre jusqu'aux logements lancés par France Télécom concernent dans un premier temps six communes des Hauts-de-Seine ainsi que cinq arrondissements de l'ouest parisien. On peut s'aventurer à dire, sans trop de risque, que le temps du déploiement de la fibre optique sur l'ensemble du territoire français sera sans commune mesure avec celui de l'ADSL, car cette dernière technologie qui recycle un réseau vieux de plusieurs décennies, ne nécessite pas de coûteux travaux de génie civil, contrairement au déploiement de la fibre optique. L'essor de la fibre optique introduit donc une nouvelle différenciation des territoires urbains dont on mesure mal l'implication sociale.

Le terme de « fracture numérique » rend improprement compte de la différentiation des territoires en fonction de la desserte en réseau de télécommunication à haut et très haut débit, dont on a vu qu'elle était d'une grande complexité. Loin de marquer une séparation claire entre deux parties de la ville, le déploiement des réseaux de télécommunications par les opérateurs introduit une multitude de niveaux de desserte des territoires, en termes de débit autorisé ou encore de qualité de service. Le terme de fragmentation urbaine, traduction de la notion de splintering urbanism développée par S. Marvin et S. Graham, rend mieux compte de la diversité des situations territoriales face aux TIC. Pour ces auteurs, cette fragmentation des quartiers en fonction de leur niveau de raccordement aux réseaux qu'a permise la libéralisation des services publics en réseaux, met en péril la cohésion urbaine qu'avaient justement forgée les réseaux urbains (viaires, électriques, d'assainissement, etc.) déployés au cours des $\mathrm{XIX}^{\mathrm{e}}$ et $\mathrm{XX}^{\mathrm{e}}$ siècles.

\section{Une fragmentation sociale par les pratiques des TIC ?}

Une première définition de la fracture numérique nous avait permis d'identifier une ligne de séparation entre les internautes et les autres. En s'intéressant aux pratiques de ceux qui utilisent Internet, on découvre, au-delà de cette distinction binaire entre les connectés et les 
non-connectés, une immense variété des modes d'utilisation des TIC. Plus qu'à partir du simple accès à Internet, on pourrait mesurer la fracture numérique à partir de la valeur d'usage d'Internet pour les individus. Or, la valeur d'usage d'Internet, c'est-à-dire son utilité mesurée en fonction de ce qu'il apporte à l'utilisateur final, en termes d'interactions sociales, d'accès à l'emploi, aux services, à l'information, etc., est elle-même grandement déterminée par l'appartenance à une catégorie sociale, l'âge, le niveau de revenu et de diplôme. Seraient ainsi du bon côté de la fracture numérique ceux qui disposent des compétences nécessaires pour utiliser les TIC de manière à atteindre leurs objectifs. Le concept de digital literacy permet d'introduire l'idée que la maîtrise des outils de télécommunications suppose l'acquisition d'un certain nombre de compétences, en somme, d'un capital intellectuel adapté aux nouveaux systèmes télécommunicationnels. Ainsi, pour E. Guichard, la fracture numérique est essentiellement d'ordre cognitif : «Avant de profiter des systèmes d'écriture contemporaine, il faut évidemment disposer d'un capital économique, pour acquérir un ordinateur et un rattachement aux réseaux ; mais aussi d'un capital social, pour se faire aider quand on ne comprend pas le (dys)fonctionnement d'un logiciel, d'un service en ligne, ou de son ordinateur; et enfin culturel, pour savoir trouver l'information que l'on recherche, et la traiter» (Guichard 2003).

On peut, en s'intéressant aux modalités d'interaction sociale sur Internet, saisir un risque de fragmentation de la société en multiples communautés virtuelles. En effet, la technologie Internet, qui consiste à relier, grâce au protocole IP, un nombre infini d'ordinateurs à travers le monde, supporte une multitude d'applications différentes (Web, email, chat, transfert de fichier, etc.), utilisant chacune des protocoles différents. Une étude des pratiques des internautes dans deux quartiers d'habitat social a révélé que les usages sont fortement déterminés par l'âge, la catégorie socio-professionnelle, ou encore, le niveau d'éducation (Beauchamps 2006). Par exemple, certains internautes négligent la messagerie électronique au profit du chat ou du blog, tandis que d'autres n'utilisent que la messagerie électronique pour échanger en ligne avec leur réseau social. De plus, la « multiplicité des applications Internet, et des communautés en ligne, tend à fragmenter les opportunités d'entrer en contact les uns avec les autres. La diversité des protocoles techniques d'échanges de messages instantanés est exemplaire de cette fragmentation: Skype, MSN, Yahoo Instant Messenger, iChat, les logiciels se multiplient sans 
qu'une adresse MSN soit capable de communiquer avec une adresse iChat $\gg^{5}$ (Beauchamps 2006, p. 26).

Les sites de réseaux sociaux offrent une illustration intéressante du risque de fragmentation sociale par les usages d'Internet. Ces dernières années ont vu se multiplier ces portails sur lesquels les internautes sont invités à créer leur profil en renseignant un certain nombre de champs comme leur âge, leur sexe, parfois leurs orientations sexuelle, politique, leurs goûts culturels, etc. Une fois mise en ligne leur identité numérique, les internautes peuvent échanger des messages avec les autres membres du portail et chercher ainsi à étendre leur réseau en créant une liste d' " amis ». Cette liste peut s'appuyer sur le réseau social préexistant de l'internaute - le portail est alors un moyen de prolonger en ligne les interactions avec les «vrais" amis - ou/et sur la mise en relation avec les autres membres du portail. La liste d'amis ainsi que les commentaires, les liens, les fichiers vidéo et sonores qui peu à peu enrichissent le profil des membres contribuent à forger l'identité numérique de l'internaute. Or, il apparaît que les sites de réseaux sociaux rassemblent des internautes dont le profil sociologique est très semblable. Aux EtatsUnis, D. Boyd a montré, à travers l'étude des pratiques d'internautes adolescents, que les deux principaux sites de réseaux sociaux américains, Myspace et Facebook, se distinguaient par l'origine sociale de leurs membres (Boyd 2007). En Belgique et en France, A.C. Orban a mis en lumière le rôle essentiel que joue, chez les jeunes internautes, la construction d'un blog pour l'identification à une communauté. Alors que Skyblog, site de réseau social sous forme de blog, domine chez la communauté des jeunes internautes, A.C. Orban récuse le terme de blogosphère pour désigner la communauté des blogueurs adultes. "L'idée d'une blogosphère unique se vide de son sens lorsque nous la confrontons à la multiplication des offres d'hébergement. La blogosphère éclate entre les différentes plateformes de blogging » (Orban 2005, p. 13). Internet produirait du même en place de l'altérité attendue, cet "entre-nous » virtuel, reproduisant la tendance à «l'entre-nous » de l'habitat urbain.

Ainsi, protocoles techniques et pratiques des internautes contribuent à fragmenter l'ensemble des internautes en multiples communautés homogènes sociologiquement. Les débats et échanges de toutes sortes

\footnotetext{
${ }^{5}$ On voit aujourd'hui apparaître des systèmes qui misent sur la compatibilité de ces différents protocoles. Le logiciel libre Adium cherche à rassembler sous une même application les multiples réseaux de messageries instantanés, repoussant ainsi un peu plus loin le risque d'éclatement des internautes en de multiples communautés.
} 
sur Internet se font essentiellement entre internautes dont le profil social et les opinions sont proches, de telle manière qu'on a pu parler d'Internet comme d'un « agent de balkanisation sociale et culturelle » (Lev-On et Manin 2006, p. 195). Il est assurément prématuré de tirer des conclusions sur d'éventuels impacts de l'essor de l'interaction sociale en ligne sur la sociabilité urbaine. Il semble cependant nécessaire de s'interroger sur les moyens offerts par Internet pour préserver sinon (ré)inventer une cohésion urbaine. Les initiatives telles que le site de réseau social www.peuplade.fr, ou le service www.toutpresdecheznous.fr, développé par la Poste, qui proposent de mettre en relation des particuliers (ou des entreprises) en vue de rencontres ou de partages de services en fonction de leur proximité spatiale illustrent le potentiel offert par Internet de mettre en relation des personnes aux profils sociologiques différents. Le site Peuplade, né en 2003 à l'initiative d'habitants du quartier des Épinettes dans le $\mathrm{XVII}^{\mathrm{e} m e}$ arrondissement parisien, se présente comme le «premier site de quartier ». Le site, qui s'est étendu à la France entière, gagne chaque mois des milliers de nouveaux membres avec un succès inégal dans les différentes villes concernées. À Paris, qui offre la densité humaine sans doute nécessaire au succès d'un tel réseau, des apéritifs de quartiers, des actions solidaires sont régulièrement organisés par les «peupladiens ». Alors que «l'apparition d'un individu "libre", affranchi de ses ancrages territoriaux, inscrits dans des réseaux de moins en moins spatialisés » (Authier et al. 2007, p. 8) a pu faire croire à la «fin des quartiers" (Ascher 1998), Internet pourrait réactiver les réseaux sociaux fondés sur la proximité géographique. Le cyberespace semble ainsi, sinon se substituer à un espace public dont on déplore régulièrement la disparition (Tomas 2001), du moins prolonger virtuellement cette « espèce d'espace » qui est la matière des villes.

\section{Le rôle des pouvoirs publics}

Face à ce double risque de fragmentation de la ville par les opérateurs de réseaux de télécommunication et de fragmentation sociale par la diversité des rapports des individus aux TIC, quel peut être le rôle des acteurs publics? Sans prétendre émettre des recommandations, on peut ici conclure en lançant des pistes de réflexions sur les moyens d'actions possibles en faveur de l'inclusion numérique. Nous avons identifié trois dimensions de la fracture numérique, terme dont nous avons admis qu'il ne rend que partiellement compte des divisions sociales et territoriales introduites par l'essor d'Internet. Une première dimension concerne l'inégalité des niveaux d'équipements des ménages en outils de télécommunication. Une deuxième dimension 
est relative à l'inégale desserte des quartiers en infrastructures de télécommunication. Enfin, la troisième dimension concerne l'inégalité des individus en matière de digital literacy, et la diversité des pratiques des TIC. Ce n'est qu'en agissant sur ces trois fronts que les acteurs publics pourront réduire les inégalités d'accès aux ressources de la société de l'information.

Les inégalités d'équipements font depuis longtemps l'objet de mesures de la part d'un grand nombre d'acteurs publics et d'associations : prêts et dons d'ordinateur, prêts bonifiés en vue de l'achat d'ordinateurs, parfois inclus dans un pack comprenant une connexion à Internet, mutualisation des connexions à Internet dans les immeubles de logements sociaux, etc.

En ce qui concerne les inégalités de desserte des quartiers en infrastructures de télécommunication, la démarche partenariale semble s'imposer. En s'associant avec les bailleurs sociaux et les opérateurs de télécommunication pour favoriser la mutualisation des réseaux de télécommunications, les collectivités territoriales peuvent permettre aux quartiers marqués par des faiblesses socio-économiques d'atteindre un niveau de desserte en infrastructure de télécommunication à la hauteur des exigences d'une société urbaine façonnée par l'émergence des TIC.

Enfin, intervenir sur la valeur d'usage d'Internet pour les individus est le volet le plus délicat et le plus ambitieux, puisqu'il nécessite d'agir dans le même temps sur des thématiques sociales plus larges que la simple inclusion numérique. Il s'agit peut-être ici d'inverser la problématique en dotant les travailleurs sociaux de compétences nouvelles, leur permettant d'inclure la dimension TIC dans l'aide qu'ils fournissent aux personnes en difficulté, notamment en raison d'un défaut d'accès aux ressources informationnelles. En s'appuyant sur les services administratifs et les structures associatives existantes, en particulier dans les quartiers défavorisés, les acteurs publics peuvent favoriser l'appropriation des TIC par les populations qui en sont éloignées ou pour lesquelles la valeur d'usage d'Internet reste faible en terme d'accès aux ressources de la société de l'information (emplois, services administratifs, commerces, santé, éducation, etc.).

\section{Bibliographie}

Alibert D., Bigot R., Foucaud D., La dynamique des inégalités en matière de nouvelles technologies. [en ligne] CREDOC, 2005, Cahier de recherche $\mathrm{n}^{\circ} 217$. Disponible sur : <http://www.credoc.fr/pdf/Rech/C217.pdf> (Consulté le 18/12/2007) 
Ascher F., La fin des quartiers? In. HAUMONT, Nicole Dir. L'urbain dans tous ces états. Faire, vivre, dire la ville, Paris, L'Harmattan, Collection Habitat et sociétés, 1998.

Authier J-Y., Bacqué M-H., Guérin-Place F., Le quartier, enjeux scientifiques, actions politiques et pratiques sociales, Paris, Editions La découverte, 2007.

Boyd D., Social Network Sites: Public, Private, or What? Knowledge Tree [Périodique en ligne] 2007, $\mathrm{n}^{\circ} 13$. Disponible sur: $<$ http://kt.flexiblelearning.net.au/tkt2007/wpcontent/uploads/2007/04/boyd.pdf> (Consulté le 18/12/07)

Brun J., Rhein C., La ségrégation dans la ville. Paris, Editions L'Harmattan, 1994.

Di Maggio P., Hargittai E., Russell Neuman W., Robinson J-P., "Social implications of the Internet". Annual Review of Sociology, 2001, vol. 27, pp. 307-36.

Dupuy G., Internet, géographie d'un réseau. Paris, Editions Ellipses, 2002.

Frydel Y., Internet au quotidien: un Français sur quatre. Insee Première. [Périodique en ligne] 2006, $\mathrm{n}^{\circ}$ 1076. Disponible sur: $<$ http://www.insee.fr/fr/ffc/ipweb/ip1076/ip1076.pdf $>$ (Consulté le 18/12/2007).

Beauchamps M., Faciliter l'accès à Internet pour les résidents de logements sociaux. [en ligne] Mémoire de Master, DDE Loire, Université de Paris 1, 2006. Disponible sur: $<\mathrm{http}: / /$ www.amenagementnumerique.net/article.php3?id_article=118> (Consulté le 18/12/2007).

Graham S., Marvin S., Splintering Urbanism: Networked Infrastructures, Technological Mobilities and the Urban Condition. London, Routledge, 2001.

Guichard É., "Does the 'Digital Divide' Exist?" In : Dir. Van Seters, P., de Gaay Fortman B. et de Ruijter A. Globalization and its new divides: malcontents, recipes, and reform, Amsterdam: Dutch University Press, 2003, pp. 69-77. ISBN : 90-361-9351-6. Traduction française [en ligne]. Disponible sur :

$<$ http://barthes.ens.fr/atelier/geo/Tilburg.html $>$ (Consulté le 18/12/07).

Huet A., Buléon P., «Collectivités territoriales, le défi du haut débit », Futuribles, 2007, n 328, pp. 51-70. 
Internet : vers une fragmentation de la ville?

Lévy J., Lussault M., Dictionnaire de la géographie et de l'espace des sociétés, Paris, Belin, 2003.

Manin B., Lev-On A., «Internet: la main invisible de la délibération », Esprit, 2006, n 324, pp. 195-212.

Orban A-C., Je blogue, tu blogues, nous bloguons. [en ligne] Étude menée pour le CLEMI, Ministère belge de l'Éducation, 2005. Disponible sur :

$<$ http://www.clemi.fr/medias_scolaires/blogs/article_blog_ACO.pdf $>$ (Consulté le 18/12/2007)

Picon A., «Les temps contrastés de l'Internet», Dir : PRELORENZO, Claude et ROUILLARD Dominique, Le temps des infrastructures, Paris, Editions l'Harmattan, 2007.

Tomas F., «L'espace public, un concept moribond ou en expansion? », Géocarrefour, 2001, vol. 76, n 1, pp. 75-84.

Sautory O., «L'accès des ménages à bas revenus aux Technologies de l'Information et de la Communication », Études et Résultats, [périodique en ligne] 2007, $\mathrm{n}^{\circ} 557$. Disponible sur: $<$ http://www.sante.gouv.fr/drees/etude-resultat/er557/er557.pdf $>$ (Consulté le 18/12/2007) 